\title{
ReaR
}

Revista electrónica de AnestesiaR

Marzo 2018

LECTURA CRÍTICA DE ARTÍCULO

\section{Ventilación mecánica en pacientes con síndrome de distrés respiratorio agudo. Lo que ya sabíamos.}

Original: Fan E, Del Sorbo L, Goligher EC, Hodgson CL, Munshi L, Walkey AJ, et al. An Official American Thoracic Society/European Society of Intensive Care Medicine/Society of Critical Care Medicine Clinical Practice Guideline: Mechanical Ventilation in Adult Patients with Acute Respiratory Distress Syndrome. Am J Respir Crit Care Med 2017;195(9):1253-1263. (Pubmed)

Monsalve Naharro JA

Complejo Hospitalario Universitario de Albacete.

\section{Resumen}

El objetivo de la ventilación mecánica (VM) en el síndrome de distrés respiratorio agudo (SDRA) consiste en asegurar el adecuado intercambio gaseoso minimizando el riesgo de lesión pulmonar. Aunque es constante el flujo de trabajos que intentan esclarecer el óptimo ajuste de los parámetros ventilatorios, su manejo en pacientes con SDRA continúa siendo un reto.

\section{Introducción}

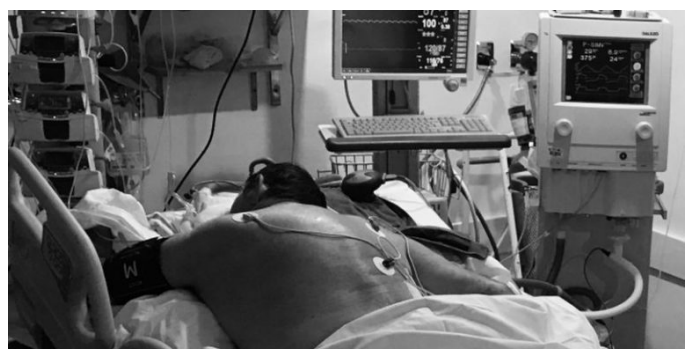

El objetivo de la ventilación mecánica (VM) en el síndrome de distrés respiratorio agudo (SDRA) consiste en asegurar el adecuado intercambio gaseoso minimizando el riesgo de lesión pulmonar. Aunque es constante el flujo de trabajos que intentan esclarecer el óptimo ajuste de los parámetros ventilatorios, su manejo en pacientes con SDRA continúa siendo un reto.

Se trata de una guía de práctica clínica con recomendaciones en el uso de estrategias ventilatorias y algunas cointervenciones en pacientes adultos con SDRA, avaladas por relevantes sociedades internacionales de Cuidados Críticos: American Thoracic Society (ATS), Society of Critical Care Medicine (SCCM), y European Society of Intensive Care Medicine (ESICM). Un panel de expertos analizó la mejor evidencia disponible abordando 6 cuestiones relacionadas con la VM: ventilación protectora (bajo volumen tidal, baja presión inspiratoria), ventilación en prono, ventilación oscilatoria de alta frecuencia, uso de presión positiva al final de la espiración (PEEP) alta o baja, uso de maniobras de reclutamiento (MR) y oxigenación por membrana extracorpórea. Las recomendaciones fueron desarrolladas aplicando el sistema GRADE (Grading of Recommendations, Assessment, Development, and Evaluation). Un resumen se expone en la Tabla 1. 


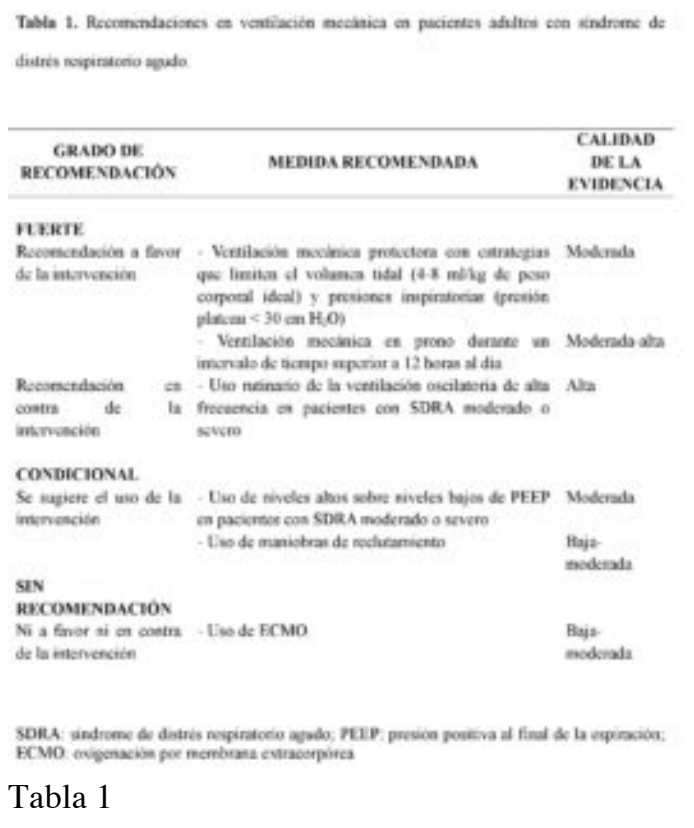

\section{Comentario}

Son limitadas las guías clínicas que exponen recomendaciones de $\mathrm{VM}$ en pacientes con SDRA (1-5), y existen numerosas controversias en cada uno de los aspectos relacionados con la terapia ventilatoria en estos pacientes. Esperábamos expectantes

recomendaciones por sociedades tan relevantes como la ESICM. Sin embargo, en nuestra opinión, no suponen nuevas aportaciones que impliquen cambios relevantes en nuestra práctica clínica diaria. Por ejemplo, en pacientes con SDRA está ampliamente aceptado el uso de una VM con una estrategia protectora para el pulmón, limitando el volumen tidal y la presión plateau. Aunque actualmente contamos con diversas estrategias ventilatorias en este sentido, la sugerida por la ARDS Network y la aproximación con pulmón abierto (Open Lung Approach) son las más ampliamente difundidas. El OLA trial (6) comparó ambas estrategias, aunque lamentablemente la inclusión de pacientes se cerró sin conseguir el tamaño muestral y no se demostraron diferencias estadísticamente significativas en la mortalidad. Quizás nos saque de dudas el ART TRIAL, que actualmente está en fase de reclutamiento.

La ventilación protectora está muy relacionada con la PEEP. Sin embargo, diversos ensayos clínicos han demostrado mejora en la supervivencia. Análisis secundarios y revisiones sistemáticas han encontrado que en pacientes con mayor severidad de la enfermedad $(\mathrm{PaO} 2 / \mathrm{FiO} 2<200)$ el uso de PEEP elevadas parece ser beneficioso y asociarse con descensos en la mortalidad. Este es el motivo por el que se sugiere con un nivel de evidencia limitado. Esta guía tampoco discute el mejor método de ajuste de la PEEP. En nuestra opinión, su titulación postreclutamiento mediante la compliance es una manera sencilla, fiable y aplicable a pie de cama del paciente, como ha sido indicado por otros autores (6).

Recientemente se ha sugerido que la driving pressure o presión de distensión es la mediadora de los efectos beneficiosos de los 3 principales componentes de la ventilación protectora: bajo volumen tidal, baja presión plateau y alta PEEP (7), si bien este hallazgo tiene que ser confirmado en adecuados ensayos clínicos aleatorizados controlados.

En la misma línea de recomendación (condicional) que el uso de PEEP elevadas se sugieren las MR (que tampoco han demostrado efectos beneficiosos sobre la mortalidad), y tampoco sin entrar en detalle en cuanto al método óptimo y el mejor momento de realización. Las MR y el ajuste de la PEEP continúan siendo uno de los aspectos de mayor controversia en VM en general y en el manejo de los pacientes con SDRA en particular, a pesar de los incontables trabajos dedicados a su estudio. Su aplicación también ha sido "sugerida" por otras guías clínicas $(1,2,5)$. Pensamos que aunque no se realicen de forma general 
y rutinaria en todos los pacientes en situación clínica estable, algunos pacientes con SDRA pueden beneficiarse de estas maniobras, especialmente los pacientes con pulmón potencialmente reclutable, incluso más que con el objetivo de mejorar la oxigenación como parte de una estrategia de protección pulmonar minimizando el atelectrauma.

Tampoco resultan relevantes las aportaciones realizadas con respecto a la ventilación en prono, difundida marcadamente tras los hallazgos del estudio PROSEVA (8). Se recomienda precozmente como terapia de primera línea en pacientes con SDRA severo. La guía de la Surviving Sepsis Campaign (1) la recomienda en pacientes con $\mathrm{PaO} 2 / \mathrm{FiO} 2<150$. En base a los resultados de los estudios publicados, se reafirma su uso prolongado ( $>12$ horas), si bien duración superiores pueden ser beneficiosas (16 horas en el estudio PROSEVA (8).

La ventilación oscilatoria de alta frecuencia parece haber quedado relegada, a pesar del entusiasmo inicial, tras los resultados de los ensayos OSCAR (9) y OSCILLATE (10) que fracasaron en mostrar beneficio en la mortalidad.

En base a la evidencia analizada, los autores de la guía no exponen recomendación a favor ni en contra de la oxigenación por membrana extracorpórea. Se trata de una técnica de difícil implantación, costosa y que requiere de una importante infraestructura. A esto se suma la presencia de complicaciones y una supervivencia no claramente superior, por lo que en espera de futuros ensayos clínicos, parece relegada como técnica de rescate en los pacientes con hipoxemia severa refractaria en los que han fracasado medidas terapéuticas menos invasivas (reclutamiento alveolar, óxido nítrico prostaglandinas inhaladas...) $(5,11)$.

Los autores también echamos en falta cuestiones no analizadas por esta guía, como por ejemplo el uso de la VM no invasiva, de determinadas modalidades ventilatorias, y de otros aspectos no relacionados con la VM como cointervenciones farmacológicas (relajación neuromuscular, corticoides), evaluación y tratamiento de la disfunción ventricular derecha, y uso de otras terapias de rescate frente a la hipoxemia refractaria.

Los autores pensamos y concluimos que aunque la estrategia ventilatoria completamente segura no existe, debería ser individualizada a cada paciente basado en su hemodinámica, intercambio gaseoso, reclutabilidad pulmonar y mecánica respiratoria.

\section{Bibliografía}

1. Rhodes A, Evans LE, Alhazzani W, Levy MM, Antonelli M, Ferrer R et al. Surviving Sepsis Campaign: International Guidelines for Management of Sepsis and Septic Shock: 2016. Intensive Care Med 2017;43(3):304-377. (PubMed) (

2. Claesson J, Freundlich M, Gunnarsson I, Laake JH, Vandvik PO, Varpula $\mathrm{T}$ et al. Scandinavian clinical practice guideline on mechanical ventilation in adults with the acute respiratory distress syndrome. Acta Anaesthesiol Scand 2015;59(3):286-97. ( $\underline{\text { PubMed) }}$ (

3. Barbas CS, Isola AM, Farias AM, Cavalcanti AB, Gama AM, Duarte AC et al. Brazilian recommendations of mechanical ventilation 2013. Part I. Rev Bras Ter Intensiva 2014;26(2):89-121. (PubMed) (HTML) (PDF) (ePDF)

4. Barbas CS, Ísola AM, Farias AM, Cavalcanti AB, Gama AM, Duarte AC et al. Brazilian recommendations of mechanical ventilation 2013. Part 2. Rev Bras Ter Intensiva 
2014;26(3):215-39. (PubMed) (HTML) (PDF) (ePDF)

5. Rodríguez A, Alvarez-Rocha L, Sirvent JM, Zaragoza R, Nieto M, Arenzana A, et al. Recommendations of the Infectious Diseases Work Group (GTEI) of the Spanish Society of Intensive and Critical Care Medicine and Coronary Units (SEMICYUC) and the Infections in Critically Ill Patients Study Group (GEIPC) of the Spanish Society of Infectious Diseases and Clinical Microbiology (SEIMC) for the diagnosis and treatment of influenza $\mathrm{A} / \mathrm{H} 1 \mathrm{~N} 1$ in seriously ill adults admitted to the Intensive Care Unit. Med Intensiva 2012;36(2):103-37. (PubMed) (HTML)

6. Kacmarek RM, Villar J, Sulemanji D, Montiel R, Ferrando C, Blanco J et al. Open Lung Approach for the Acute Respiratory Distress Syndrome: A Pilot, Randomized Controlled Trial. Crit Care Med 2016;44(1):3242. (PubMed)

7. Amato MB, Meade MO, Slutsky AS, Brochard L, Costa EL, Schoenfeld DA et al. Driving pressure and survival in the acute respiratory distress syndrome. $\mathrm{N}$ Engl J Med 2015;372(8):747-55. (PubMed) (HTML) (PDF)

8. Guérin C, Reignier J, Richard JC, Beuret P, Gacouin A, Boulain $\mathrm{T}$ et al. Prone Positioning in Severe Acute Respiratory Distress Syndrome. N Engl J Med 2013;368(23):2159-68. (PubMed) (HTML) (PDF)
9. Young D, Lamb SE, Shah S, MacKenzie I, Tunnicliffe W, Lall R et al. High-frequency oscillation for acute respiratory distress syndrome. N Engl J Med 2013;368(9):80613. (PubMed) (

10. Ferguson ND, Cook DJ, Guyatt GH, Mehta S, Hand L, Austin P et al. High-frequency oscillation in early acute respiratory distress syndrome. N Engl J Med 2013;368(9):795805. (PubMed) (HTML) ( $\underline{\text { PDF }}$

11. Richard C, Argaud L, Blet A, Boulain T, Contentin L, Dechartres A et al. Extracorporeal life support for patients with acute respiratory distress syndrome: report of a Consensus Conference. Ann Intensive Care 2014;4:15. ( PubMed) (ePub) (PDF)

Correspondencia al autor

Jose Ángel Monsalve Naharro jose_mn@hotmail.com

FEA Servicio de Anestesiología, Reanimación y Terapéutica del Dolor.

Complejo Hospitalario Universitario de Albacete.

Publicado en AnestesiaR el 6 de septiembre de 2017 